RHIC/AP/17

\title{
Beam emittance dilution going through profile measurement flags
}

\author{
Ping Zhou \\ December 1, 1993
}

\section{Introduction}

Phosphor screens will be used in the AGS to RHIC transfer line to measure beam transverse profiles. This is very important in the sextant test for diagnostics and matching. With multiple flags, 3 minimum, beam transverse emittance can be measured for each bunch. Possibilities also exist to use them during normal operation to monitor beam quality during injection. However, as the beam goes through these flags, multiple scattering with the material will increase the angular spread of the beam and cause emittance dilution. The magnitude of this dilution may very well limit the total number of flags that may be used in one measurement. This note will estimate the magnitude of emittance dilution.

\section{Multiple scattering and emittance dilution}

The bulk of the deflection as charged particles traverse a medium is due to elastic Coulomb scattering from the nuclei within the medium. The distribution of the deflections is roughly Gaussian for small deflections while having greater probability for large-angle scattering[1]. For our purpose we can use a Gaussian distribution

$$
f(\theta)=\exp \left(-\frac{\theta}{2 \sigma^{2}}\right)
$$


with the rms angular spread being[1]:

$$
\sigma=\frac{14.1 M \epsilon V / c}{p \beta} Z_{i} \sqrt{\frac{L}{X_{0}}} \times\left(1+\frac{1}{9} \log _{10} \frac{L}{X_{0}}\right)
$$

where $p, \beta$ and $Z$ are the momentum, relative velocity and charge number of the beam particles, $L$ is the thickness of the medium and $X_{0}$ is the radiation length of the medium material.

Assuming the beam is bi-Gaussian in the phase space with Twiss parameters $\alpha, \beta, \gamma$ and rms emittance $\epsilon$, the phase space distribution is

$$
\rho\left(x, x^{\prime}\right)=N \exp \left[-\frac{1}{2 \epsilon}\left(\gamma x^{2}+2 \alpha x x^{\prime}+3 x^{2}\right)\right]
$$

with

$$
3 \gamma-\alpha^{2}=1
$$

The normalization constant $N$ is not important here since we do not include any particle loss. and will be used to stand for any constant needed.

The beam phase space distribution after passing through the medium is thus the convolution of the original distribution with the scattering angle distribution:

$$
\begin{aligned}
\rho^{\prime}\left(x, x^{\prime}\right) & =\int \rho(x, t) \times f\left(x^{\prime}-t\right) d t \\
& =N \int \exp \left[-\frac{1}{2 \epsilon}\left(\gamma x^{2}+2 \alpha x t+\beta t^{2}\right)\right] \times \exp \left[-\frac{\left(x^{\prime}-t\right)^{2}}{2 \sigma^{2}}\right] d t \\
& =N \exp \left(-\frac{\gamma x^{2}}{2 \epsilon}-\frac{x^{\prime 2}}{2 \sigma^{2}}\right) \times \exp \left[\frac{1}{2} \frac{\left(\frac{x^{\prime}}{\sigma^{2}}-\frac{\alpha}{\epsilon} x\right)^{2}}{\left(\frac{\beta}{\epsilon}+\frac{1}{\sigma^{2}}\right)}\right] \\
& =N \exp \left[-\frac{\left(\frac{\gamma}{\epsilon}+\frac{\sigma^{2}}{\epsilon^{2}}\right) x^{2}+2 \frac{\alpha}{\epsilon} x x^{\prime}+\frac{\beta}{\epsilon} x^{\prime 2}}{2\left(1+\frac{\beta \sigma^{2}}{\epsilon}\right)}\right] \\
& =\exp \left[-\frac{\gamma^{\prime} x^{2}+2 \alpha^{\prime} x x^{\prime}+\beta^{\prime} x^{\prime 2}}{2 \epsilon^{\prime}}\right]
\end{aligned}
$$

where $\alpha^{\prime}, \beta^{\prime}, \gamma^{\prime}$ and $\epsilon^{\prime}$ are the Twiss parameters and emittance of the beam after passing through the flag. Since the Twiss parameters satisfy

$$
\beta^{\prime} \gamma^{\prime}-\alpha^{\prime 2}=1
$$


the new emittance can thus be obtained

$$
\begin{aligned}
\epsilon^{r 2} & =\frac{\left(1+\frac{\beta \sigma^{2}}{\epsilon}\right)^{2}}{\frac{3}{\epsilon}\left(\frac{\gamma}{\epsilon}+\frac{\sigma^{2}}{\epsilon^{2}}\right)-\left(\frac{\alpha}{\epsilon}\right)^{2}} \\
& =\epsilon^{2} \times\left(1+\frac{\beta \sigma^{2}}{\epsilon}\right)
\end{aligned}
$$

which leads to

$$
\epsilon^{\prime}=\epsilon \sqrt{1+\frac{\beta \sigma^{2}}{\epsilon}}
$$

In the limit of small angular deflection

$$
\Delta \epsilon \approx \epsilon^{\prime}-\epsilon=\frac{\beta \sigma^{2}}{2}
$$

As can be seen above. the shape and orientation of the phase space ellipse is slightly changed after going through a flag. The new Twiss parameters are:

$$
\begin{aligned}
& \alpha^{\prime}=\frac{\alpha}{\sqrt{1+\beta \sigma^{2} / \epsilon}} \\
& \beta^{\prime}=\frac{\beta}{\sqrt{1+\beta \sigma^{2} / \epsilon}} \\
& \gamma^{\prime}=\frac{\gamma+\sigma^{2} / \epsilon}{\sqrt{1+\beta \sigma^{2} / \epsilon}}
\end{aligned}
$$

The emittance dilution is proportional to the $\beta$-function value at the flag. This result is expected, at least qualitatively, since thin flags only deflect the beam particles, and the emittance increase is proportional to the beam size given the fixed angular divergence.

\section{AGS to RHIC transfer line}

The flags to be used in the transfer line consist of a 2 mil thick of $G_{2} \mathrm{O}_{2} S: T b$ phosphor screen on a 1mil thick of aluminum plate[2]. The phosphor screen material was described in[3] as having a density of about $3 \mathrm{~g} / \mathrm{cm}^{3}$ and a radiation length of $8.5 \mathrm{~g} / \mathrm{cm}^{2}$. For aluminum the density is $2.7 \mathrm{~g} / \mathrm{cm}^{3}$ and the 
radiation length is $24 \mathrm{~g} / \mathrm{cm}^{2}$ [4]. Since the monitor is placed at a $45^{\circ}$ angle with the beam, the thicknesses of the phosphor and the aluminum base seen by the beam are thus $22 \mathrm{mg} / \mathrm{cm}^{2}$ and $9.7 \mathrm{mg}: \mathrm{cm}^{2}$ respectively. The total thickness of the flag measured in radiation lengths is thus

$$
\frac{L}{X_{0}}=\left(\frac{L}{X_{0}}\right)_{p h}+\left(\frac{L}{X_{0}}\right)_{a i}=0.003
$$

RMS deflection angles are calculated for gold ion and proton beams. Parameters[5] and results for the two beams are listed in Table 1.

\begin{tabular}{|c|c|c|c|cc|}
\hline Beam & $\mathrm{Z}$ & $\mathrm{A}$ & $\gamma$ & $\epsilon(\mathrm{mm} \cdot \mathrm{mrad}$ & $\sigma$ ( $\mu$ radian $)$ \\
\hline $\mathrm{Au}$ & 79 & 197 & 12.6 & 0.13 & 19 \\
\hline Proton & 1 & 1 & 31.2 & 0.11 & 19 \\
\hline
\end{tabular}

Table 1: Beam Parameters at injection and scattering angle width

The current transfer line design has several locations that flags may be placed. The locations and the related parameters are listed in Table 2, along with the calculated emittance increase for each flag. Since proton and gold ion beam suffer the same angular divergence through the flags, they also get the same absolute emittance growth listed here.

\begin{tabular}{|c|c|c|c|c|}
\hline Flag location & $\beta_{x}(\mathrm{~m})$ & $\beta_{y}(\mathrm{~m})$ & $\Delta \epsilon_{x}(\mathrm{~mm} \cdot \mathrm{mrad})$ & $\Delta \epsilon_{y}(\mathrm{~mm} \cdot \mathrm{mrad})$ \\
\hline UFLAG1 & 63.2 & 3.8 & 0.011 & 0.00069 \\
\hline UF2 & 6.4 & 6.3 & 0.0012 & 0.0011 \\
\hline UF3 & 11.6 & 61.2 & 0.0021 & 0.011 \\
\hline UFLAG4 & 52.5 & 11.9 & 0.0095 & 0.0021 \\
\hline UFLAG5 & 12.2 & 52.9 & 0.0022 & 0.0095 \\
\hline WFLAG1 & 65.8 & 6.4 & 0.012 & 0.0012 \\
\hline WFLAG2 & 5.9 & 93.7 & 0.0011 & 0.017 \\
\hline WFLAG3 & 100 & 8.7 & 0.018 & 0.0016 \\
\hline
\end{tabular}

Table 2: List of flags and their associated beam emittance growths 
The results above show clearly that we cannot indiscriminately use multiple flags for emittance measurements. It might be possible to use a few flags with small emittance dilution plus any flag as the last one in the measurement. There are two complications associated with this approach. however. One is that this requires different sets of flags to measure emittances in the horizontal and vertical direction. The other is that among the possible selections, betatron phase advances between the Hags may be inadequate for emittance measurements. More studies are needed to find the best configuration for beam emittance measurement.

\section{References}

[1] Thomas G. Trippe. Physics Vade Mecum, chapter 9. page 160. American Institute of Physics, 2nd edition, 1989.

[2] R. Witkover. Private communication.

[3] D.P. Russell and K.T. McDonald. A beam-profile monitor for the bnl accelerator test facility (ATF). In Proceedings of the 1989 IEEE Particle Accelerator Conference, pages 1510-1512, March 1989.

[4] Thomas G. Trippe. Physics Vade Mecum, chapter 9, page 165. American Institute of Physics, 2nd edition, 1989.

[5] RHIC Design Manual, February 1992. 